\title{
Fuzzy Covering based Rough Sets Revisited
}

\author{
Lynn D'eer ${ }^{1}$ Chris Cornelis ${ }^{1,2}$ Daniel Sánchez ${ }^{2,3}$ \\ ${ }^{1}$ Department of Applied Mathematics, Computer Science and Statistics, Ghent University, Belgium. \\ ${ }^{2}$ Department of Computer Science and Artificial Intelligence, University of Granada, Spain. \\ ${ }^{3}$ European Centre for Soft Computing, Edificio Científico-Tecnológico, Asturias, Spain
}

\begin{abstract}
In this paper we review four fuzzy extensions of the socalled tight pair of covering based rough set approximation operators. Furthermore, we propose two new extensions of the tight pair: for the first model, we apply the technique of representation by levels to define the approximation operators, while the second model is an intuitive extension of the crisp operators. For the six models, we study which theoretical properties they satisfy. Moreover, we discuss interrelationships between the models.
\end{abstract}

Keywords: rough sets, fuzzy sets, covering, approximation operators

\section{Introduction}

To analyze incomplete information, Pawlak [1] proposed the theory of rough sets, which has a widespread application in artificial intelligence, data analysis and pattern recognition. Originally, Pawlak designed his model using an equivalence relation to describe the indiscernibility between objects. This way, a set can be approximated by a lower and upper approximation. The former consists of equivalence classes which are included in the set, while the latter consists of equivalence classes which have a non-empty intersection with the set. However, the use of an equivalence relation may be too strict for the applications. Therefore, many generalizations of Pawlak's model which use coverings instead of a partition have been presented. The first authors proposing covering based rough sets were Żakowski [2] and Pomykała [3]. Yao and Yao presented an extensive survey on covering based rough sets in 2012 [4].

During the last decades, not only the research of rough set models flourished. Hybridizations of rough set theory and fuzzy set theory introduced by Zadeh [5] have been a trending topic in many fields. Several authors have proposed fuzzy covering based rough set models $[6,7,8,9,10]$. In this paper, we discuss four models which are all extensions of the so-called tight pair of covering based rough set approximation operators [11]. Moreover, we introduce two new extensions of the tight pair. We study theoretical properties of the models, as well as interrelationships between the models.

The outline of the paper is as follows: in Section 2, we recall the basic concepts of covering based rough sets and fuzzy sets and a non-nested level-based representation of fuzzy concepts. In Section 3, we discuss six fuzzy covering based rough set models which all extend the tight covering based rough set model from a theoretical point of view. Moreover, in Section 4, we study interrelationships between the six models. Conclusions are stated in Section 5.

\section{Preliminaries}

In this section, we recall basic notions on covering based rough sets, fuzzy sets and the technique of representation by levels. We will assume that the universe $U$ is a finite, non-empty set.

\subsection{Covering based Rough Sets}

In 1982, Pawlak introduced rough set theory to deal with uncertainty due to incompleteness and indiscernibility in information systems [1]. An equivalence relation $E$ on the universe of discourse $U$ is used to describe the indiscernibility between the elements of the universe. Based on the relation $E$, the lower and upper approximation operators of a subset $A \subseteq U$ are defined. The former contains all the elements certainly belonging to the set $A$, while the latter contains the elements possibly belonging to $A$.

Definition 1. [1] Let $E$ be an equivalence relation on $U$. The lower and upper approximation operator based on $E$, denoted by $\operatorname{apr}_{E}$ and $\overline{\mathrm{apr}}_{E}$, respectively, are defined as follows, for $\overline{A \subseteq} U$ :

$$
\begin{aligned}
\underline{\operatorname{apr}}_{E}(A) & =\left\{x \in U \mid[x]_{E} \subseteq A\right\} \\
& =\bigcup\left\{[x]_{E} \in U / E \mid[x]_{E} \subseteq A\right\}, \\
\overline{\operatorname{apr}}_{E}(A) & =\left\{x \in U \mid[x]_{E} \cap A \neq \emptyset\right\} \\
& =\bigcup\left\{[x]_{E} \in U / E \mid[x]_{E} \cap A \neq \emptyset\right\},
\end{aligned}
$$

where $[x]_{E}$ represents the equivalence class of $x$ by $E$ and the quotient $U / E$ the set of equivalence classes.

Equations (2) and (4) are sometimes called the granule based definitions of Pawlak's rough set model [4]. For an equivalence relation $E$, the equivalence classes $[x]_{E}$ form a partition of the universe $U$. A generalization of Pawlak's model is obtained when the partition is replaced by a covering:

Definition 2. Let $\mathbb{C}=\left\{K_{i} \subseteq U \mid K_{i} \neq \emptyset, i \in I\right\}$ be a collection of non-empty subsets of $U$ for a finite index set $I$, then $\mathbb{C}$ is called a covering if $\cup_{i \in I} K_{i}=U$. Moreover, the ordered pair $(U, \mathbb{C})$ is called a covering approximation space. 
Żakowski [2] was the first to define a straightforward generalization of the granule based definitions of Pawlak's model, by replacing the partition $U / E$ by a more general covering $\mathbb{C}$. However, the lower and upper approximation operators defined by Żakowski were not dual to each other. Therefore, Pomykała [3] adopted the definitions of Żakowski and introduced their dual operators:

Definition 3. [3] Let $(U, \mathbb{C})$ be a covering approximation space. Two pairs of dual approximation operators $\left(\underline{\operatorname{apr}_{\mathbb{C}}^{\prime}}, \overline{\operatorname{apr}}_{\mathbb{C}}^{\prime}\right)$ and $\left(\underline{\operatorname{apr}}_{\mathbb{C}}^{\prime \prime}, \overline{\operatorname{apr}}_{\mathbb{C}}^{\prime \prime}\right)$ are defined as follows:

$$
\begin{aligned}
\underline{\operatorname{apr}}_{\mathbb{C}}^{\prime}(A) & =\bigcup\{K \in \mathbb{C} \mid K \subseteq A\} \\
& =\{x \in U \mid(\exists K \in \mathbb{C})(x \in K \wedge K \subseteq A)\}, \\
\overline{\operatorname{apr}}_{\mathbb{C}}^{\prime}(A) & =\operatorname{co}\left(\underline{\operatorname{apr}} \mathbb{C}^{\prime}(\operatorname{co}(A))\right) \\
& =\{x \in U \mid(\forall K \in \mathbb{C})(x \in K \Rightarrow K \cap A \neq \emptyset)\}, \\
\underline{\operatorname{apr}}_{\mathbb{C}}^{\prime \prime}(A) & =\operatorname{co}\left(\overline{\operatorname{apr}}_{\mathbb{C}}^{\prime \prime}(\operatorname{co}(A))\right) \\
& =\{x \in U \mid(\forall K \in \mathbb{C})(x \in K \Rightarrow K \subseteq A)\}, \\
\overline{\operatorname{apr}}_{\mathbb{C}}^{\prime \prime}(A) & =\bigcup\{K \in \mathbb{C} \mid K \cap A \neq \emptyset\} \\
& =\{x \in U \mid(\exists K \in \mathbb{C})(x \in K \wedge K \cap A \neq \emptyset)\},
\end{aligned}
$$

for $A \subseteq U$ and co the set-theoretic complement.

The pair $\left(\operatorname{apr}_{\mathbb{C}}^{\prime}, \overline{\operatorname{apr}}_{\mathbb{C}}^{\prime}\right)$ is often called the tight pair of covering based approximation operators, and $\left(\operatorname{apr}_{\mathbb{C}}^{\prime \prime}, \overline{\operatorname{apr}}_{\mathbb{C}}^{\prime \prime}\right)$ is called the loose pair [11].

In this paper, we focus on the fuzzy extensions of the tight pair $\left(\operatorname{apr}_{\mathbb{C}}^{\prime}, \overline{\operatorname{apr}}_{\mathbb{C}}^{\prime}\right.$ ). Since we no longer use an equivalence relaton to define the operators, not all properties of the model of Pawlak are satisfied in the covering approximation space $(U, \mathbb{C})$. For example, it no longer holds that $\operatorname{apr}_{\mathbb{C}}^{\prime}(A \cap B)=\operatorname{apr}_{\mathbb{C}}^{\prime}(A) \cap \operatorname{apr}_{\mathbb{C}}^{\prime}(B)$ for $A, B \subseteq U$. The properties which are satisfied by the approximation operators $\operatorname{apr}_{\mathbb{C}}^{\prime}$ and $\overline{\operatorname{apr}}_{\mathbb{C}}^{\prime}$ are listed in Table 1 . Note that $\mathbb{C} \subseteq \mathbb{C}^{\prime}$ if and only if $\forall K \in \mathbb{C}: K \in \mathbb{C}^{\prime}$.

\subsection{Fuzzy Set Theory}

Fuzzy set theory was introduced by Zadeh [5] in 1965 in order to model vagueness in information systems.

Definition 4. [5] A fuzzy set $A$ in the universe $U$ is a mapping $A: U \rightarrow[0,1]$. The set of fuzzy sets of $U$ is denoted by $\mathscr{F}(U)$. Given $\alpha \in[0,1]$, the weak $\alpha$-level set of $A$ is the crisp set $A_{\alpha}=\{x \in U \mid A(x) \geq \alpha\}$. Moreover, for $\alpha \in[0,1]$, the (fuzzy) constant $\alpha$-set $\hat{\alpha}$ is defined by $\hat{\alpha}(x)=\alpha$ for all $x \in U$.

In the crisp case, the only constant $\alpha$-sets are $\hat{0}=\emptyset$ and $\hat{1}=U$. Below we discuss fuzzy logical connectives which are the fuzzy extension of the Boolean ones.

Definition 5. A conjunctor $\mathscr{C}$ is a mapping $\mathscr{C}:[0,1]^{2} \rightarrow$ $[0,1]$ which is increasing in both arguments and satisfies the border conditions of the Boolean conjunction. It is called a border conjunctor if $\mathscr{C}(1, a)=a$ for all $a \in[0,1]$. A commutative and associative border conjunctor is called a $t$-norm and is denoted by $\mathscr{T}$.
An implicator $\mathscr{I}$ is a mapping $\mathscr{I}:[0,1]^{2} \rightarrow[0,1]$ which is decreasing in the first argument and increasing in the second, and which satisfies the border conditions of the Boolean implication. An implicator is called border if $\mathscr{I}(1, a)=$ a for all $a \in[0,1]$.

Furthermore, a negator $\mathscr{N}$ is a decreasing mapping $\mathscr{N}:[0,1] \rightarrow[0,1]$ such that $\mathscr{N}(0)=1$ and $\mathscr{N}(1)=$ 0 . A negator is called involutive if for all $a \in[0,1]$, $\mathscr{N}(\mathscr{N}(a))=a$. The standard negator is defined by $\mathscr{N}_{S}(a)=1-$ a for $a \in[0,1]$.

Given a negator $\mathscr{N}$, the $\mathscr{N}$-complement of a fuzzy set $A$ is the fuzzy set $\operatorname{co}_{\mathscr{N}}(A)$ defined by, for $x \in U$, $\left(\operatorname{co}_{\mathscr{N}}(A)\right)(x)=\mathscr{N}(A(x))$.

In order to extend the rough set models to the fuzzy setting, we recall some notions of fuzzy relations and fuzzy coverings.

Definition 6. A fuzzy relation $R \in \mathscr{F}(U \times U)$ is called serial, if $(\forall x \in U)(\exists y \in U)(R(x, y)=1)$ and it is called reflexive if $(\forall x \in U)(R(x, x)=1)$. Given a fuzzy relation $R$, the foreset $R x$ of an element $x$ is a fuzzy set in $U$ defined as follows: $\forall y \in U: R x(y)=R(y, x)$.

In literature, different definitions of fuzzy coverings are proposed in $[6,12]$. However, we will use the following one, where the condition $\cup \mathbb{C}=U$ is maintained for infinite coverings.

Definition 7. Let I be an (infinite) index set. A collection $\mathbb{C}=\left\{K_{i} \in \mathscr{F}(U) \mid K_{i} \neq \emptyset, i \in I\right\}$ is called a fuzzy covering, if for all $x \in U$ there exists a $K \in \mathbb{C}$ such that $K(x)=1$. If $\mathbb{C}$ is a fuzzy covering, then the ordered pair $(U, \mathbb{C})$ is called a fuzzy covering approximation space.

Note that the set $\{R x \mid x \in U\}$ for $R$ a serial fuzzy relation is a fuzzy covering.

\subsection{Technique of Representation by Levels}

In 2012, Sánchez et al. [13] introduced a non-nested level-based representation of fuzziness. The idea is to describe a fuzzy concept with crisp representatives, each one being a crisp realization under a certain condition [13]. Different levels of restriction are considered, with the levels in $[0,1]$, where level 1 is the most restrictive level. Level 0 represents no restriction at all, but it will not be taken into account in the representation. Since humans can only distinguish a finite set of levels, for each fuzzy concept it is assumed that there exists a finite set of levels $\mathscr{A}=\left\{\alpha_{1}, \alpha_{2}, \ldots, \alpha_{m}\right\}$ with $1=\alpha_{1}>\alpha_{2}>\ldots>\alpha_{m}>\alpha_{m+1}=0$ and $m \geq 1$.

A fuzzy concept $A$ is described by a representation by levels (RL) $\left(\Lambda_{A}, \rho_{A}\right)$ if $\Lambda_{A}$ is a finite set of levels and $\rho_{A}: \Lambda_{A} \rightarrow \mathscr{P}(A)$ is a function which projects each level $\alpha$ on a crisp subset of $A$. The set of crisp representatives $\Omega_{A}$ of $\operatorname{RL}\left(\Lambda_{A}, \rho_{A}\right)$ is given by $\Omega_{A}=\left\{\rho_{A}(\alpha) \mid \alpha \in \Lambda_{A}\right\}$. Note that a fuzzy set $A$ can be seen as a special case of RL: let $\Lambda_{A}=\{A(x) \mid A(x)>0\} \cup\{1\}$ and $\rho_{A}(\alpha)=A_{\alpha}$ for each $\alpha \in \Lambda_{A}$.

The crisp representatives on each level are independent of each other. Moreover, they are not necessarily nested, i.e., $\alpha>\beta \nRightarrow \rho(\alpha) \supseteq \rho(\beta)$. 


\begin{tabular}{lll}
\hline (D) & Duality & $\underline{\operatorname{apr}}_{\mathbb{C}}^{\prime}(A)=\operatorname{co}\left(\overline{\operatorname{apr}}_{\mathbb{C}}^{\prime}(\operatorname{co}(A))\right)$ \\
\hline (INC) & Inclusion & $\underline{\operatorname{apr}}_{\mathbb{C}}^{\prime}(A) \subseteq A \subseteq \overline{\operatorname{apr}}_{\mathbb{C}}^{\prime}(A)$ \\
\hline (SM) & Set Monotonicity & $A \subseteq B \Rightarrow \underline{\operatorname{apr}}_{\mathbb{C}}^{\prime}(A) \subseteq \underline{\operatorname{apr}}_{\mathbb{C}}^{\prime}(B), \overline{\operatorname{apr}}_{\mathbb{C}}^{\prime}(A) \subseteq \overline{\operatorname{apr}}_{\mathbb{C}}^{\prime}(B)$ \\
\hline (CM) & Covering Monotonicity & $\mathbb{C} \subseteq \mathbb{C}^{\prime} \Rightarrow \underline{\operatorname{apr}}_{\mathbb{C}}^{\prime}(A) \subseteq \underline{\operatorname{apr}}_{\mathbb{C}^{\prime}}^{\prime}(A), \overline{\operatorname{apr}}_{\mathbb{C}^{\prime}}^{\prime}(A) \subseteq \overline{\operatorname{apr}}_{\mathbb{C}}^{\prime}(A)$ \\
\hline (ID) & Idempotence & $\underline{\operatorname{apr}}_{\mathbb{C}}^{\prime}\left(\underline{\operatorname{apr}}_{\mathbb{C}}^{\prime}(A)\right)=\underline{\operatorname{apr}}_{\mathbb{C}}^{\prime}(A), \overline{\operatorname{apr}}_{\mathbb{C}}^{\prime}\left(\overline{\operatorname{apr}}_{\mathbb{C}}^{\prime}(A)\right)=\overline{\operatorname{apr}}_{\mathbb{C}}^{\prime}(A)$ \\
\hline (UE) & Universe and Empty Set & $\underline{\operatorname{apr}}_{\mathbb{C}}^{\prime}(U)=U=\overline{\operatorname{apr}}_{\mathbb{C}}^{\prime}(U), \underline{\operatorname{apr}}_{\mathbb{C}}^{\prime}(\emptyset)=\emptyset=\overline{\operatorname{apr}}_{\mathbb{C}}^{\prime}(\emptyset)$ \\
\hline
\end{tabular}

Table 1: Properties of $\left(\underline{\operatorname{apr}}_{\mathbb{C}}^{\prime}, \overline{\operatorname{apr}}_{\mathbb{C}}^{\prime}\right)$ for the covering approximation space $(U, \mathbb{C})$

Although this technique is useful to represent fuzzy information, it is not easy to interpret by humans. Therefore, it is possible to obtain a fuzzy set that summarizes the information given by the RL: let $\left(\Lambda_{A}, \rho_{A}\right)$ be an RL associated with a fuzzy concept $A$, then the fuzzy summary $v_{A}: U \rightarrow[0,1]$ is given by

$$
\begin{aligned}
v_{A}(x) & =\sum_{Y \in \Omega_{A} \mid x \in Y}\left(\sum_{\alpha_{i} \mid Y=\rho_{A}\left(\alpha_{i}\right)}\left(\alpha_{i}-\alpha_{i+1}\right)\right) \\
& =\sum_{\alpha_{i} \in \Lambda_{A} \mid x \in \rho_{A}\left(\alpha_{i}\right)}\left(\alpha_{i}-\alpha_{i+1}\right) .
\end{aligned}
$$

Considering operations on fuzzy concepts, this technique will allow to perform the associated crisp operations on each level of the RL. Let $f: \mathscr{P}(U)^{n} \rightarrow \mathscr{P}(U)$ be a crisp operation, then $f$ is extended to RLs in the following way: let $\left(A_{1}, A_{2}, \ldots, A_{n}\right)$ be fuzzy concepts in $U$ with for each $A_{i}$ an $\operatorname{RL}\left(\Lambda_{A_{i}}, \rho_{A_{i}}\right)$, then $f\left(A_{1}, A_{2}, \ldots, A_{n}\right)$ is a fuzzy concept in $U$ represented by $\left(\Lambda_{f\left(A_{1}, A_{2}, \ldots, A_{n}\right)}, \rho_{f\left(A_{1}, A_{2}, \ldots, A_{n}\right)}\right)$ where

$$
\Lambda_{f\left(A_{1}, A_{2}, \ldots, A_{n}\right)}=\bigcup_{1 \leq i \leq n} \Lambda_{A_{i}}
$$

and $\forall \alpha \in \Lambda_{f\left(A_{1}, A_{2}, \ldots, A_{n}\right)}$,

$$
\rho_{f\left(A_{1}, A_{2}, \ldots, A_{n}\right)}(\alpha)=f\left(\rho_{A_{1}}(\alpha), \rho_{A_{2}}(\alpha), \ldots, \rho_{A_{n}}(\alpha)\right) .
$$

Therefore, we have the following proposition:

Proposition 1. [13] Operations on RLs satisfy all the properties of the Boolean logic.

In other words, all properties that hold for a crisp concept, will also hold for its fuzzification, when RLs are used. This is the main advantage of non-nested levelbased representations.

\section{Extensions of $\left(\underline{\operatorname{apr}}_{\mathbb{C}}^{\prime}, \overline{\operatorname{apr}}_{\mathbb{C}}^{\prime}\right)$}

In this section, we study different fuzzy covering based rough set models which extend the crisp operators $\left(\operatorname{apr}_{\mathbb{C}}^{\prime}, \overline{\operatorname{apr}}_{\mathbb{C}}^{\prime}\right)$. That is, if the covering $\mathbb{C}$ and the approximated set $A$ are crisp, the fuzzy covering based approximation operators coincide with $\left(\operatorname{apr}_{\mathbb{C}}^{\prime}, \overline{\operatorname{apr}}_{\mathbb{C}}^{\prime}\right)$.

For each model, we discuss which properties of Table 1 are maintained. Note that in a fuzzy setting, the property (UE) is extended to the property (CS):

$$
\text { (CS) } \forall \alpha \in[0,1]: \underline{\operatorname{apr}}_{\mathbb{C}}^{\prime}(\hat{\alpha})=\hat{\alpha}=\overline{\operatorname{apr}}_{\mathbb{C}}^{\prime}(\hat{\alpha}) .
$$

Some authors specified the fuzzy logical connectives they used in their model, while others used a general t-norm $\mathscr{T}$ and an implicator $\mathscr{I}$. In order to obtain a relevant comparison, we will study the properties of the models for the following choices of logical connectives if applicable:

- A left-continuous t-norm $\mathscr{T}$ and its R-implicator $\mathscr{I}_{\mathscr{T}}$ defined by, for $a, b \in[0,1]$,

$$
\mathscr{I}_{\mathscr{T}}(a, b)=\sup \{c \in[0,1] \mid \mathscr{T}(a, c) \leq b\} .
$$

The couple $\left(\mathscr{T}, \mathscr{I}_{\mathscr{T}}\right)$ satisfies the residuation principle [14]:

$$
\forall a, b, c \in[0,1]: \mathscr{T}(a, b) \leq c \Leftrightarrow \mathscr{I}_{\mathscr{T}}(a, c) \geq b .
$$

- The minimum t-norm $\mathscr{T}_{M}(a, b)=\min (a, b)$ and the Kleene-Dienes implicator $\mathscr{I}_{K D}(a, b)=\max (1-$ $a, b)$, for $a, b \in[0,1]$.

\subsection{Model of Li et al.}

The first fuzzy covering based rough set model we discuss, was introduced by Li et al. in 2008 [6].

Definition 8. [6] Let $(U, \mathbb{C})$ be a fuzzy covering approximation space, $\mathscr{T}$ a t-norm and $\mathscr{I}$ an implicator, then the pair of fuzzy operators $\left(\underline{\operatorname{apr}}_{\mathbb{C}, \mathrm{Li}}, \overline{\mathrm{apr}}_{\mathbb{C}, \mathrm{Li}}\right.$ ) is defined as follows: let $A \in \mathscr{F}(U), x \in U$,

$$
\begin{aligned}
& \left(\underline{\operatorname{apr}_{\mathbb{C}, \mathrm{Li}}},(A)\right)(x)=\sup _{K \in \mathbb{C}} \mathscr{T}\left(K(x), \inf _{y \in U} \mathscr{I}(K(y), A(y))\right), \\
& \left(\overline{\operatorname{apr}}_{\mathbb{C}, \mathrm{Li}}^{\prime}(A)\right)(x)=\inf _{K \in \mathbb{C}} \mathscr{I}\left(K(x), \sup _{y \in U} \mathscr{T}(K(y), A(y))\right) .
\end{aligned}
$$

This model was proposed by the authors to define a more general model than the model discussed in [15], where the covering $\mathbb{C}=\{R x \mid x \in U\}$ was used, for a serial fuzzy relation $R$.

Next, we discuss the properties of this model for the cases $\left(\mathscr{T}, \mathscr{I}_{\mathscr{T}}\right)$ and $\left(\mathscr{T}_{M}, \mathscr{I}_{K D}\right)$. Note that originally, $\mathrm{Li}$ et al. considered a continuous t-norm and its R-implicator.

\subsubsection{Left-continuous t-norm and its R-implicator}

Assume $(\mathscr{T}, \mathscr{I})=\left(\mathscr{T}, \mathscr{I}_{\mathscr{T}}\right)$ with $\mathscr{T}$ left-continuous. 
Proposition 2. Let $\mathscr{N}$ be an involutive negator. If for all $x \in U, \mathscr{I}_{\mathscr{T}}(x, 0)=\mathscr{N}(x)$, then $\left(\underline{\operatorname{apr}^{\prime}}{ }_{\mathbb{C} . \mathrm{Li}}, \overline{\operatorname{apr}}_{\mathbb{C}, \mathrm{Li}}\right)$ satisfies (D) with respect to $\mathscr{N}$. Furthermore, $\left(\underline{\mathrm{apr}^{\prime}, \mathrm{Li}}, \overrightarrow{\mathrm{apr}}_{\mathbb{C}, \mathrm{Li}}^{\prime}\right)$ satisfies (INC), (SM), (CM) and (ID).

Proof. (D), (INC), (SM) and (ID) are proved in [6], while (CM) follows directly from the definition.

Proposition 3. The pair $\left(\underline{\operatorname{apr}}_{\mathbb{C}, \mathrm{Li}}^{\prime}, \overline{\operatorname{apr}}_{\mathbb{C}, \mathrm{Li}}^{\prime}\right)$ satisfies $(C S)$.

Proof. Take $\alpha \in[0,1]$. Since (INC) holds, it is sufficient to prove that $\operatorname{apr}_{\mathbb{C}, \mathrm{Li}}^{\prime}(\hat{\alpha}) \supseteq \hat{\alpha}$ and $\overline{\operatorname{apr}}_{\mathbb{C}, \mathrm{Li}}(\hat{\alpha}) \subseteq \hat{\alpha}$. Let $x \in U$ and $K^{*} \in \mathbb{C}$ such that $K^{*}(x)=1$, then

$$
\begin{aligned}
& \left(\underline{\operatorname{apr}^{\prime}, \mathrm{Li}}\right. \\
& =\mathscr{T}\left(1, \inf _{y \in U} \mathscr{I}\left(K^{*}(y), \alpha\right)\right)=\inf _{y \in U} \mathscr{I}\left(K^{*}(y), \alpha\right) \\
& \geq \inf _{y \in U} \mathscr{I}(1, \alpha)=\alpha,
\end{aligned}
$$

where we have used that $K^{*}(y) \leq 1$ for all $y \in U$, and the fact that an R-implicator is border [14].

The proof for $\overline{\operatorname{apr}}_{\mathbb{C} . \mathrm{Li}}(\hat{\alpha}) \subseteq \hat{\alpha}$ is analogous.

Note that in [6], (CS) was stated given that the covering $\mathbb{C}$ was normalized, i.e., $(\forall K \in \mathbb{C})(\exists x \in U)(K(x)=$ $1)$. This condition was necessary, since Li et al. defined a covering $\mathbb{C}$ as follows: let $\mathbb{C}$ be a family of non-empty fuzzy sets in $U$, then it is a covering if $\cup \mathbb{C}=U$. However, in this case it is not guaranteed that $(\forall x \in U)(\exists K \in$ $\mathbb{C})(K(x)=1)$.

\subsubsection{Minimum t-norm and Kleene-Dienes implicator}

Assume $(\mathscr{T}, \mathscr{I})=\left(\mathscr{T}_{M}, \mathscr{I}_{K D}\right)$

Proposition 4. The pair $\left(\underline{\operatorname{apr}}_{\mathbb{C}, \mathrm{Li}}^{\prime}, \overrightarrow{\mathrm{apr}}_{\mathbb{C}, \mathrm{Li}}^{\prime}\right)$ satisfies $(D)$ with respect to $\mathscr{N}_{S}$ and the operators satisfy (SM) and (CM) as well.

Proof. Straightforward from the definitions.

The properties (INC), (ID), (CS) and (UE) do not hold.

Example 1. Let $U=\{x, y\}, \mathbb{C}=\left\{K_{1}, K_{2}, K_{3}\right\}$ with $K_{1}(x)=1$ and $K_{1}(y)=0.6, K_{2}(x)=0.4$ and $K_{2}(y)=$ $1, K_{3}(x)=0.2$ and $K_{3}(y)=0.7$. Take $A=U$, then $\left(\overline{\operatorname{apr}}_{\mathbb{C}, \mathrm{Li}}(A)\right)(x)=0.8$ and $\left(\overline{\operatorname{apr}}_{\mathbb{C}, \mathrm{Li}}^{\prime}(A)\right)(y)=0.7$. Hence, $A \nsubseteq \overline{\operatorname{apr}}_{\mathbb{C}, \mathrm{Li}}^{\prime}(A)$. Thus, (INC), (UE) and (CS) do not hold. Furthermore, (ID) does not hold, since

$$
\left(\overline{\operatorname{apr}}_{\mathbb{C}, \mathrm{Li}}\left(\overline{\operatorname{apr}}_{\mathbb{C}, \mathrm{Li}}(A)\right)\right)(x)=0.7 \neq\left(\overline{\operatorname{apr}}_{\mathbb{C}, \mathrm{Li}}^{\prime}(A)\right)(x) .
$$

\subsection{Model of Feng et al.}

The next model we discuss, is the model of Feng et al. [7]. Feng et al. used the induced covering of a fuzzy covering $\mathbb{C}$. Given a covering $\mathbb{C}$, then for each $x \in U$, let

$$
K_{x}=\cap\{K \in \mathbb{C} \mid K(x)=1\},
$$

then $\operatorname{Cov}(\mathbb{C})=\left\{K_{x} \mid x \in U\right\}$ is a fuzzy covering of $U$ as well, called the induced covering of $\mathbb{C}$.
Definition 9. [7] Let $(U, \mathbb{C})$ be a fuzzy covering approximation space, then the pair of fuzzy operators $\left(\underline{\operatorname{apr}^{\prime}, F e}, \overrightarrow{\operatorname{apr}}_{\mathbb{C}, \mathrm{Fe}}\right)$ is defined as follows:

$$
\begin{aligned}
& \left(\underline{\operatorname{apr}_{\mathbb{C}, \mathrm{Fe}}^{\prime}}(A)\right)(x)=\inf _{y \in U} \max \left(1-K_{x}(y), A(y)\right), \\
& \left(\overline{\operatorname{apr}}_{\mathbb{C}, \mathrm{Fe}}^{\prime}(A)\right)(x)=\sup _{y \in U} \min \left(K_{x}(y), A(y)\right),
\end{aligned}
$$

for $A \in \mathscr{F}(U), x \in U$ and $K_{x}$ as defined in Equation (5).

This model is very much alike to the relation based fuzzy rough set model of Dubois and Prade $[16,17]$. Indeed, if for all $x \in U$ the fuzzy set $K_{x}$ of the induced covering $\operatorname{Cov}(\mathbb{C})$ is equal to the foreset $R x$ for a reflexive relation $R$, then the model of Dubois and Prade is obtained.

Note that Feng et al. specifically use the minimum tnorm and the Kleene-Dienes implicator. Therefore, it is only useful to study the properties of the model for these fuzzy logical connectives.

Proposition 5. The pair $\left(\underline{\operatorname{apr}}_{\mathbb{C}, \mathrm{Fe}}^{\prime}, \overline{\mathrm{apr}}_{\mathbb{C}, \mathrm{Fe}}^{\prime}\right)$ satisfies $(D)$ with respect to $\mathscr{N}_{S}$ and the properties (INC), (SM), (CM) and (CS). It satisfies (ID) if and only if

$$
\forall x, z \in U: \sup _{y \in U} \min \left(K_{x}(y), K_{y}(z)\right)=K_{x}(z) .
$$

Proof. The properties (D), (INC), (SM), (CM) and (CS) are proved in [7]. The proof that property (ID) is satisfied, given the condition, is pursued from the following observations:

$$
\begin{aligned}
& \left.\underline{\underline{\operatorname{apr}}} \underset{\mathbb{C}, F e}{\prime} \underline{\operatorname{apr}^{\prime}} \underline{C}, \mathrm{Fe}^{\prime}(A)\right)(x) \\
& =\inf _{z \in U} \max \left(1-\sup _{y \in U} \min \left(K_{x}(y), K_{y}(z)\right), A(z)\right)
\end{aligned}
$$

and

$$
\begin{aligned}
& \left(\overline{\operatorname{apr}}_{\mathbb{C}, \mathrm{Fe}}^{\prime}\left(\overline{\operatorname{apr}}_{\mathbb{C}, \mathrm{Fe}}^{\prime}(A)\right)\right)(x) \\
& =\sup _{z \in U} \min \left(\sup _{y \in U} \min \left(K_{x}(y), K_{y}(z)\right), A(z)\right)
\end{aligned}
$$

\subsection{Model of Inuiguchi et al.}

Next, we study the model of Inuiguchi et al. [8, 9]. They used the following logical connective: let $\mathscr{I}$ be an implicator, then $\xi[\mathscr{I}]:[0,1]^{2} \rightarrow[0,1]$ is defined by

$\forall a, b \in[0,1]: \xi[\mathscr{I}](a, b)=\inf \{c \in[0,1] \mid \mathscr{I}(a, c) \geq b\}$

$\xi[\mathscr{I}]$ is a conjunctor if $\forall a \in[0,1[: \xi[\mathscr{I}](1, a)<1[9]$.

Furthermore, note that $\mathscr{I}$ needs to be upper semicontinuous, which is the same as to require that $\mathscr{I}$ is left-continuous in the first parameter and rightcontinuous in the second, in order to have the following equivalence [8]:

$$
\forall a, b, c \in[0,1]: \xi[\mathscr{I}](a, b) \leq c \Leftrightarrow \mathscr{I}(a, c) \geq b .
$$

The model of Inuiguchi et al. is given in the following definition: 
Definition 10. [8, 9] Let $(U, \mathbb{C})$ be a fuzzy covering approximation space, $\mathscr{I}$ an upper semi-continuous implicator and $\mathscr{N}$ an involutive negator, then the pair of fuzzy operators $\left(\underline{\operatorname{apr}}{ }_{\mathbb{C}, \text { In }}, \overrightarrow{\operatorname{apr}}_{\mathbb{C}, \text { In }}\right)$ is defined as follows: let $A \in \mathscr{F}(U), x \in U$,

$\left.\underline{\operatorname{apr}_{\mathbb{C}, \text { In }}}(A)\right)(x)=\sup _{K \in \mathbb{C}} \xi[\mathscr{I}]\left(K(x), \inf _{y \in U} \mathscr{I}(K(y), A(y))\right)$,

$\left(\overline{\operatorname{apr}}_{\mathbb{C}, \text { In }}^{\prime}(A)\right)(x)=\operatorname{co}_{\mathscr{N}}\left(\underline{\operatorname{apr}}_{\mathbb{C}, \text { In }}^{\prime}\left(\operatorname{co}_{\mathscr{N}}(A)\right)\right)(x)$.

In [8] and [9], a collection $\mathscr{F} \subseteq \mathscr{F}(U)$ was used to define the operators. However, we will always assume that the collection $\mathscr{F}$ is a covering.

In order to study the properties, we assume that $\mathscr{I}$ is either the R-implicator of a left-continuous t-norm, or the Kleene-Dienes implicator.

\subsubsection{Left-continuous $t$-norm and its $R$-implicator}

Assume $\mathscr{I}=\mathscr{I}_{\mathscr{T}}$ for a left-continuous t-norm $\mathscr{T}$. Note that every R-implicator is upper semi-continuous [8].

In this case, the model of Inuiguchi et al. coincides with the model of $\mathrm{Li}$ et al. defined in Definition 8. To see this, we prove the following proposition:

Proposition 6. Let $\mathscr{T}$ be a left-continuous t-norm and $\mathscr{I}$ its $R$-implicator, then $\xi\left[\mathscr{I}_{\mathscr{T}}\right]=\mathscr{T}$.

Proof. Take $a, b \in[0,1]$, then

$$
\begin{aligned}
\xi\left[\mathscr{I}_{\mathscr{T}}\right](a, b) & =\inf \left\{c \in[0,1] \mid \mathscr{I}_{\mathscr{T}}(a, c) \geq b\right\} \\
& =\inf \{c \in[0,1] \mid \mathscr{T}(a, b) \leq c\} \\
& =\mathscr{T}(a, b),
\end{aligned}
$$

since $\left(\mathscr{T}, \mathscr{I}_{\mathscr{T}}\right)$ satisfies the residuation principle.

Therefore, we can conclude the following

Corollary 1. Let $\mathscr{T}$ be a left-continuous t-norm and $\mathscr{I}_{\mathscr{T}}$ its $R$-implicator, then

$$
\left(\underline{\operatorname{apr}}_{\mathbb{C}, \text { In }}^{\prime}, \overline{\operatorname{apr}}_{\mathbb{C}, \text { In }}^{\prime}\right)=\left(\underline{\operatorname{apr}}_{\mathbb{C}, \mathrm{Li}}^{\prime}, \overline{\operatorname{apr}}_{\mathbb{C}, \mathrm{Li}}^{\prime}\right) .
$$

Hence, the properties of $\left(\underline{\operatorname{apr}}_{\mathbb{C}, \text { In }}^{\prime}, \overline{\operatorname{apr}}_{\mathbb{C}, \text { In }}^{\prime}\right)$ in the case of an R-implicator based on a left-continuous t-norm can be obtained from the results in Section 3.1.1.

\subsubsection{Minimum t-norm and Kleene-Dienes implicator}

Assume $\mathscr{I}=\mathscr{I}_{K D}$, then

$$
\xi\left[\mathscr{I}_{K D}\right](a, b)=\left\{\begin{array}{ll}
0 & 1-a \geq b \\
b & 1-a<b
\end{array} .\right.
$$

Note that $\xi\left[\mathscr{I}_{K D}\right]$ is a border conjunctor, but not a tnorm. The Kleene-Dienes implicator is upper semicontinuous [8].

Proposition 7. The pair $\left(\underline{\operatorname{apr}^{\prime}},{ }_{\mathbb{C}, I n}, \overrightarrow{\operatorname{apr}}_{\mathbb{C}, \text { In }}^{\prime}\right)$ satisfies $(D)$, (INC), (SM), (CM) and (ID).
Proof. Properties (D), (INC), (SM) and (ID) are proved in [8] and [9], (CM) follows from the definitions.

Furthermore, the (CS) property is fulfilled.

Proposition 8. The pair $\left.\underline{\operatorname{apr}}_{\mathbb{C}, I n}^{\prime}, \overline{\operatorname{apr}}_{\mathbb{C}, \text { In }}^{\prime}\right)$ satisfies (CS).

Proof. We will only prove (CS) for the lower approximation operator, since $\left(\underline{\operatorname{apr}^{\prime}}{ }_{\mathbb{C} \text {,In }}, \overline{\operatorname{apr}}_{\mathbb{C} \text {,In }}^{\prime}\right)$ are dual. Let $\alpha \in[0,1]$ and take $x \in U$. As $\mathbb{C}$ is a covering, let $K^{*} \in \mathbb{C}$ with $K^{*}(x)=1$. Since (INC) holds, we only need to prove that $\left(\underline{\operatorname{apr}}_{\mathbb{C}, I n}^{\prime}(\hat{\alpha})\right)(x) \geq \alpha$ :

$$
\begin{aligned}
& \left(\underline{\operatorname{apr}_{\mathbb{C}, \text { In }}^{\prime}}(\hat{\alpha})\right)(x) \geq \xi\left[\mathscr{I}_{K D}\right]\left(K^{*}(x), \inf _{y \in U} \mathscr{I}_{K D}\left(K^{*}(y), \alpha\right)\right) \\
& =\xi\left[\mathscr{I}_{K D}\right]\left(1, \inf _{y \in U} \max \left(1-K^{*}(y), \alpha\right)\right) \\
& =\inf _{y \in U} \max \left(1-K^{*}(y), \alpha\right) \geq \inf _{y \in U} \alpha=\alpha .
\end{aligned}
$$

Hence, (CS) is satisfied.

\subsection{Model of Wu et al.}

The next model we discuss is introduced by $\mathrm{Wu}$ et al. [10] in 2012. It is inspired by the use of weak $\alpha$ level sets for $K \in \mathbb{C}$.

Definition 11. [10] Let $(U, \mathbb{C})$ be a fuzzy covering approximation space, then the pair of fuzzy operators $\left(\underline{\operatorname{apr}}_{\mathbb{C}, \mathrm{Wu}}, \overline{\operatorname{apr}}_{\mathbb{C}, \mathrm{Wu}}\right)$ is defined as follows: let $A \in \mathscr{F}(U)$, $x \in U$,

$$
\begin{aligned}
& \left.\underline{\underline{\operatorname{apr}}}_{\mathbb{C}, \mathrm{Wu}}(A)\right)(x)=\sup _{K \in \mathbb{C}} \inf \{A(y) \mid K(y) \geq K(x), y \in U\}, \\
& \left(\overline{\operatorname{apr}}_{\mathbb{C}, \mathrm{Wu}}(A)\right)(x)=\inf _{K \in \mathbb{C}} \sup \{A(y) \mid K(y) \geq K(x), y \in U\} .
\end{aligned}
$$

As with the model of Feng et al., Wu et al. did not use fuzzy logical connectives to define the model. The properties are discussed in the next proposition.

Proposition 9. The operators $\underline{\operatorname{apr}}_{\mathbb{C}, \mathrm{Wu}}$ and $\overline{\mathrm{apr}}_{\mathbb{C}, \mathrm{Wu}}$ satisfy (D) with respect to $\mathscr{N}_{S}$ and they satisfy (INC), (SM), $(C M),(I D)$ and $(C S)$.

Proof. Properties (D), (INC), (SM), (CM) and (CS) are stated in [10]. It is straightforward that (CS) also holds.

\subsection{Representation by Levels}

A possible way to construct a fuzzy extension of the crisp operator $\operatorname{apr}_{\mathbb{C}}^{\prime}$, is to apply the technique of representation by levels stated in Section 2.3:

Definition 12. Let $(U, \mathbb{C})$ be a fuzzy covering approximation space and $A$ a fuzzy set. The fuzzy set $\underline{\operatorname{apr}_{\mathbb{C}, \mathrm{RBL}}}(A)$ is represented by the $R L$ $\left(\Lambda_{\underline{\text { apr }}_{\mathbb{C}, \mathrm{RBL}}^{\prime}}(A), \rho_{\underline{\text { apr }}_{\mathbb{C}, \mathrm{RBL}}^{\prime}}(A)\right)$, with

$$
\begin{aligned}
\Lambda_{\underline{\text { apr }}_{\mathbb{C}, \mathrm{RBL}}^{\prime}(A)} & =\Lambda_{A} \cup \Lambda_{\mathbb{C}}, \\
\rho_{\underline{\mathrm{apr}}_{\mathbb{C}, \mathrm{RBL}}^{\prime}(A)}^{\prime}(\alpha) & =\cup\left\{K_{\alpha} \mid K \in \mathbb{C}, K_{\alpha} \subseteq A_{\alpha}\right\},
\end{aligned}
$$


for all $\alpha \in \Lambda_{\mathrm{apr}_{\mathbb{C}, \mathrm{RBL}}}(A)$. To obtain the membership degree of $x$ in $\operatorname{apr}_{\mathbb{C}, \mathrm{RBL}}(A)$, we compute the fuzzy summary:

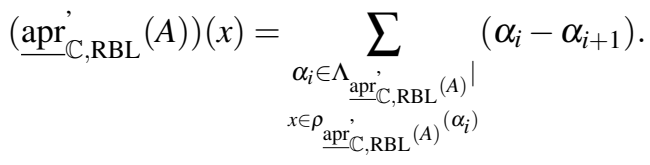

The upper approximation operator $\overrightarrow{\operatorname{apr}}_{\mathbb{C}, \mathrm{RBL}}(A)$ is obtained in a similar way, by taking

$$
\rho_{\overline{\mathrm{apr}_{\mathbb{C}, \mathrm{RBL}}}}(A)=\operatorname{co}\left(\rho_{\underline{\mathrm{apr}}_{\mathbb{C}, \mathrm{RBL}}^{\prime}}(\operatorname{co}(A))\right)
$$

for each $\alpha \in \Lambda_{\text {apr }_{\mathbb{C}, \mathrm{RBL}}^{\prime}(A)}$.

It is clear that for a crisp set $A$ and a crisp covering $\mathbb{C}$, the crisp sets $\left(\operatorname{apr}_{\mathbb{C}}^{\prime}(A), \overline{\operatorname{apr}}_{\mathbb{C}}^{\prime}(A)\right)$ are obtained.

By construction, the model satisfies all properties:

Proposition 10. The pair ( $\left.\underline{\mathrm{apr}}_{\mathbb{C}, \mathrm{RBL}}^{\prime}, \overline{\mathrm{apr}}_{\mathbb{C}, \mathrm{RBL}}^{\prime}\right)$ satisfies (D), (INC), (SM), (CM), (ID) and (UE).

Proof. Immediate consequence of Proposition 1.

\subsection{Intuitive Extension}

The final model we discuss is an intuitive extension of $\left(\operatorname{apr}_{\mathbb{C}}^{\prime}, \overline{\operatorname{apr}}_{\mathbb{C}}^{\prime}\right)$. The lower approximation operator is obtained by replacing the union by the supremum, and by taking the membership degrees of $x$ into account. We introduce it in the following definition:

Definition 13. Let $(U, \mathbb{C})$ be a fuzzy covering approximation space and $\mathscr{N}$ an involutive negator, then the pair of fuzzy operators $\left(\underline{\operatorname{apr}}_{\mathbb{C} \text {.InEx }}, \overline{\operatorname{apr}}_{\mathbb{C}, \mathrm{InEx}}\right)$ is defined as follows: let $A \in \mathscr{F}(U), x \in U$,

$$
\begin{aligned}
& \left.\underline{\left(\operatorname{apr}_{\mathbb{C}, \text { InEx }}^{\prime}\right.}(A)\right)(x)=\sup _{K \in \mathbb{C}}\{K(x) \mid K \subseteq A\} \\
& \left(\overline{\operatorname{apr}}_{\mathbb{C}, \operatorname{InEx}}^{\prime}(A)\right)(x)=\operatorname{co}_{\mathscr{N}}\left(\underline{\operatorname{apr}}{ }_{\mathbb{C}, \operatorname{InEx}}^{\prime}\left(\operatorname{co}_{\mathscr{N}}(A)\right)\right)(x) .
\end{aligned}
$$

It is clear that for a crisp covering $\mathbb{C}$ and a crisp set $A$ the pair $\left(\underline{\operatorname{apr}}_{\mathbb{C}}^{\prime}, \overline{\operatorname{apr}}_{\mathbb{C}}^{\prime}\right)$ is obtained. A drawback of this model is that it is quite extreme: if $\forall K \in \mathbb{C}: K \nsubseteq A$ for a given $A \in \mathscr{F}(U)$, then $\operatorname{apr}_{\mathbb{C}, \text { InEx }}(A)=\emptyset$.

We study its properties in the following proposition.

Proposition 11. The pair $\left(\underline{\operatorname{apr}_{\mathbb{C} . \mathrm{InEx}},}, \overrightarrow{\mathrm{apr}}_{\mathbb{C}, \mathrm{InEx}}\right)$ satisfies (D), (INC), (SM), (CM), (ID) and (UE).

Proof. The properties (D), (INC), (SM) and (CM) follow immediately from the definition. (ID) follows from the fact that $K \subseteq \underline{\operatorname{apr}}_{\mathbb{C}, \mathrm{InEx}}(A) \Leftrightarrow K \subseteq A$. Finally, (UE) holds since for $x \in U,\left(\underline{\operatorname{apr}}_{\mathbb{C}, \operatorname{InEx}}(\emptyset)\right)(x)=\sup \emptyset=0$ and $\left.\underline{\operatorname{apr}_{\mathbb{C}, \operatorname{InEx}}^{\prime}}(U)\right)(x) \geq K^{*}(x)=1$, with $K^{*} \in \mathbb{C}$ such that $K^{*}(x)=1$.

Although $\left(\underline{\text { apr }_{\mathbb{C}, I n E x}^{\prime}}, \overrightarrow{\operatorname{apr}}_{\mathbb{C}, \text { InEx }}^{\prime}\right)$ satisfies (UE), it does not satisfy $(\mathrm{CS})$ :

Example 2. Let $U=\{x, y\}$ and $\mathbb{C}=\left\{K_{1}, K_{2}\right\}$ with $K_{1}(x)=K_{2}(y)=1$ and $K_{1}(y)=K_{2}(x)=0.3$. Let $A=\hat{\alpha}$ with $\alpha=0.4$, then neither $K_{1}$ nor $K_{2}$ is included in $A$. Hence, $\left(\underline{\operatorname{apr}}_{\mathbb{C}, \operatorname{InEx}}^{\prime}(A)\right)(x)=\sup \emptyset=0$.

\subsection{Summary of the Properties}

In Table 2, we give an overview of the properties for the different fuzzy covering based rough set model discussed in Section 3. The symbol $\checkmark$ indicates that the property is satisfied, while the symbol $\boldsymbol{X}$ indicates that the property is not satisfied. If $t \boldsymbol{x}$ is denoted, then the property is satisfied given a certain condition.

The properties (SM) and (CM) are satisfied by all models. The model of Inuiguchi et al. with the use of the Kleene-Dienes implicator and the model using the technique of representation by levels satisfy all properties without extra conditions. The model of Li et al. with the connectives $\left(\mathscr{T}_{M}, \mathscr{I}_{K D}\right)$ is not preferable from theoretic point of view, since it does not satisfy (INC), (ID), (CS) and (UE).

\section{Interrelationships between Fuzzy Extensions of $\left(\underline{\operatorname{apr}}_{\mathbb{C}}^{\prime}, \overline{\operatorname{apr}}_{\mathbb{C}}^{\prime}\right)$}

In this section, we study different interrelationships between the models stated in Section 3. We discuss whether the approximation operators of the different models are comparable. We restrict ourselves to the discussion of the lower approximation operators. Due to duality, similar results can be obtained for the upper approximation operators. Two lower approximation operators are comparable, if one can be included in the other. Again we will distinguish between $\left(\mathscr{T}, \mathscr{I}_{\mathscr{T}}\right)$ with $\mathscr{T}$ left-continuous and $\left(\mathscr{T}_{M}, \mathscr{I}_{K D}\right)$, if applicable.

Firstly, the lower approximations of the intuitive extension, the model based on representation by levels and the model of $\mathrm{Wu}$ et al. are comparable as stated in the following two propositions:

Proposition 12. Let $\mathbb{C}$ be a covering and $A \in \mathscr{F}(U)$, then $\underline{\text { apr }^{\prime}} \mathbb{C}, \mathrm{InEx}(A) \subseteq \underline{\text { apr }_{\mathbb{C}, \mathrm{RBL}}^{\prime}}(A)$.

Proof. If there is no $K \in \mathbb{C}$ with $K \subseteq A$, then $\underline{\operatorname{apr}}_{\mathbb{C} \text {.InEx }}(A)=\emptyset$, thus the inclusion holds.

On the other hand, take $K \in \mathbb{C}$ with $K \subseteq A$, then for all $\alpha \in[0,1], K_{\alpha} \subseteq A_{\alpha}$. We need to prove that for $x \in U$, $K(x) \leq\left(\underline{\operatorname{apr}}_{\mathbb{C}, \mathrm{RBL}}(A)\right)(x)$.

If $K(x)=0$, then the inclusion holds, so assume $K(x)=\gamma$ with $\gamma \neq 0$. For all $\alpha \leq \gamma$ it holds that $K(x) \geq \alpha$, hence $x \in K_{\alpha}$. Since $K_{\alpha} \subseteq A_{\alpha}$, we obtain

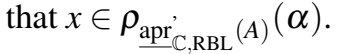

Let $\Lambda_{\text {apr }_{\mathbb{C}, \mathrm{RBL}}^{\prime}}(A)=\left\{\alpha_{1}, \alpha_{2}, \ldots, \alpha_{m}\right\}$ with $\alpha_{i}>\alpha_{i+1}$ for all $1 \leq i \leq m$ and $\alpha_{m+1}=0$. Since $K(x)=\gamma$, $\gamma \in \Lambda_{\text {apr }_{\mathbb{C}, \mathrm{RBL}}^{\prime}}(A)$. Therefore, there exists a $1 \leq i \leq m$ such that $\gamma=\alpha_{i}$. By the fact that $x \in \rho_{\underline{\text { apr }}_{\mathbb{C}, \mathrm{RBL}}(A)}(\alpha)$ for all $\alpha \leq \gamma$ and the definition of fuzzy summary, we obtain that

$$
\begin{aligned}
& \left.\underline{\operatorname{apr}}_{\mathbb{C}, \mathrm{RBL}}^{\prime}(A)\right)(x) \\
& \geq\left(\alpha_{i}-\alpha_{i+1}\right)+\left(\alpha_{i+1}-\alpha_{i+2}\right)+\ldots+\left(\alpha_{m}-\alpha_{m+1}\right) \\
& =\alpha_{i}=\gamma
\end{aligned}
$$

Therefore, $K(x) \leq\left(\underline{\operatorname{apr}_{\mathbb{C}}, \mathrm{RBL}}(A)\right)(x)$. 


\begin{tabular}{|c|c|c|c|c|c|c|c|}
\hline & (D) & (INC) & (SM) & $(\mathrm{CM})$ & (ID) & (CS) & (UE) \\
\hline Li et al., Inuiguchi et al. $\left(\mathscr{T}, \mathscr{I}_{\mathscr{T}}\right)$ & $i$ & $\checkmark$ & $\checkmark$ & $\checkmark$ & $\checkmark$ & $\checkmark$ & $\checkmark$ \\
\hline Li et al. $\left(\mathscr{T}_{M}, \mathscr{I}_{K D}\right)$ & is & $x$ & $\checkmark$ & $\checkmark$ & $x$ & $x$ & $x$ \\
\hline Feng et al. & is & $\checkmark$ & $\checkmark$ & $\checkmark$ & is & $\checkmark$ & $\checkmark$ \\
\hline Inuiguchi et al. $\mathscr{I}_{K D}$ & $\checkmark$ & $\checkmark$ & $\checkmark$ & $\checkmark$ & $\checkmark$ & $\checkmark$ & $\checkmark$ \\
\hline Wu et al. & $i x$ & $\checkmark$ & $\checkmark$ & $\checkmark$ & $\checkmark$ & $\checkmark$ & $\checkmark$ \\
\hline Representation by Levels & $\checkmark$ & $\checkmark$ & $\checkmark$ & $\checkmark$ & $\checkmark$ & $\checkmark$ & $\checkmark$ \\
\hline Intuitive Extension & $\checkmark$ & $\checkmark$ & $\checkmark$ & $\checkmark$ & $\checkmark$ & $x$ & $\checkmark$ \\
\hline
\end{tabular}

Table 2: Overview of the properties for the fuzzy approximation space $(U, \mathbb{C})$

Proposition 13. Let $\mathbb{C}$ be a covering and $A \in \mathscr{F}(U)$, then $\underline{\operatorname{apr}^{\prime}}{ }_{\mathbb{C}, \mathrm{RBL}}(A) \subseteq \underline{\operatorname{apr}^{\prime}} \mathbb{C}, \mathrm{Wu}^{(A)}(A$

Proof. Take $x \in U$. If there is no $\alpha \in \Lambda_{\text {apr }_{\mathbb{C} \text { RBL }}^{\prime}}(A)$ such that $x \in \rho_{\underline{\text { apr }}}^{\prime}{ }_{\mathbb{C}, \mathrm{RBL}}(A)(\alpha)$, then $\left.\underline{\operatorname{apr}}_{\mathbb{C}, \mathrm{RBL}}^{\prime}(A)\right)(x)=0$ and thus, $\left(\underline{\operatorname{apr}}_{\mathbb{C}, \mathrm{RBL}}^{\prime}(A)\right)(x) \leq\left(\underline{\operatorname{apr}}_{\mathbb{C}, \mathrm{Wu}}^{\prime}(A)\right)(x)$.

On the other hand, let $\alpha^{*} \in \Lambda_{\text {apr }_{\mathbb{C}, \mathrm{RBL}}^{\prime}(A)}$ be the largest level such that $x \in \rho_{\text {apr }_{\mathbb{C}, \mathrm{RBL}}(A)}\left(\alpha^{*}\right)$. We will prove that $\left(\underline{\operatorname{apr}^{\prime}, \mathrm{RBL}^{\prime}}(A)\right)(x) \leq \alpha^{*}$ and $\alpha^{*} \leq\left(\underline{\operatorname{apr}^{\prime}, \mathrm{Cu}}(A)\right)(x)$.

For the first inequality, note that $\left(\underline{\operatorname{apr}}_{\mathbb{C}, \mathrm{RBL}}(A)\right)(x)$ reaches its maximum if $x \in \rho_{\text {apr }_{\mathbb{C}, \text { RBL }}^{\prime}(A)}(\alpha)$ for all $\alpha \leq$ $\alpha^{*}$, i.e., if $x$ is in all crisp representatives for the levels $\alpha \leq \alpha^{*}$. Denote $\Lambda_{\text {apr }_{\mathbb{C}, \mathrm{RBL}}^{\prime}(A)}=\left\{\alpha_{1}, \alpha_{2}, \ldots, \alpha_{m}\right\}$ with $\alpha_{i}>\alpha_{i+1}$ for all $1 \leq i \leq m$ and $\alpha_{m+1}=0$. Then there exists a $1 \leq i \leq m$ such that $\alpha^{*}=\alpha_{i}$. Hence,

$$
\begin{aligned}
& \left.\underline{\operatorname{apr}}_{\mathbb{C}, \mathrm{RBL}}(A)\right)(x) \\
& \leq\left(\alpha_{i}-\alpha_{i+1}\right)+\left(\alpha_{i+1}-\alpha_{i+2}\right)+\ldots+\left(\alpha_{m}-\alpha_{m+1}\right) \\
& =\alpha_{i}=\alpha^{*} .
\end{aligned}
$$

For the second inequality, since $x \in \rho_{\underline{\mathrm{apr}}_{\mathbb{C}, \mathrm{RBL}}(A)}\left(\alpha^{*}\right)$, there exists a $K^{*} \in \mathbb{C}$ with $K_{\alpha^{*}} \subseteq A_{\alpha^{*}}$ and $x \in K_{\alpha^{*}}^{*}$. Moreover, for every $y \in U$ with $K^{*}(y) \geq K^{*}(x)$, it holds that $y \in K_{\alpha^{*}}^{*}$, and thus $y \in A_{\alpha^{*}}$. Therefore,

$$
\begin{aligned}
& \left.\underline{\operatorname{apr}}_{\mathbb{C}, \mathrm{Wu}}^{\prime}(A)\right)(x) \geq \inf _{y \in U}\left\{A(y) \mid K^{*}(y) \geq K^{*}(x)\right\} \\
& \geq \inf _{y \in U}\left\{\alpha^{*} \mid K^{*}(y) \geq K^{*}(x)\right\}=\alpha^{*} .
\end{aligned}
$$

This completes the proof.

Secondly, the model of Feng et al. is incomparable with the model of $\mathrm{Wu}$ et al., the model based on representation by levels and the intuitive extension.

Finally, for comparing the models of $\mathrm{Li}$ et al. and Inuiguchi et al. to these four models, we distinguish between $\left(\mathscr{T}, \mathscr{I}_{\mathscr{T}}\right)$ with $\mathscr{T}$ left-continuous and $\left(\mathscr{T}_{M}, \mathscr{I}_{K D}\right)$.

\subsection{Left-continuous t-norm and its R-implicator}

In this case, the model of Inuiguchi et al. coincides with the model of Li et al., so we only discuss comparability with the latter one. The model of Li et al. is comparable with the intuitive extension:

Proposition 14. Let $\mathbb{C}$ be a covering and $A \in \mathscr{F}(U)$, then for a left-continuous $\mathscr{T}$ and its $R$-implicator it holds that $\underline{\text { apr }}{ }_{\mathbb{C}, \operatorname{InEx}}(A) \subseteq \underline{\operatorname{apr}_{\mathbb{C}, \mathrm{Li}}}{ }^{(}(A)$.

Proof. Define $\mathbb{C}^{\prime}=\{K \in \mathbb{C} \mid K \subseteq A\} \subseteq \mathbb{C}$, then for all $K \in \mathbb{C}^{\prime}$ it holds that $\inf _{y \in U} \mathscr{I}_{\mathscr{T}}(K(y), A(y))=1$ (see [14]). Therefore, we obtain for $x \in U$ that

$$
\begin{aligned}
& \left.\underline{\underline{\operatorname{apr}}}_{\mathbb{C}, \mathrm{Li}}^{\prime}(A)\right)(x) \geq \sup _{K \in \mathbb{C}^{\prime}} \mathscr{T}\left(K(x), \inf _{y \in U} \mathscr{I}_{\mathscr{T}}(K(y), A(y))\right) \\
& =\sup _{K \in \mathbb{C}^{\prime}} \mathscr{T}(K(x), 1)=\sup \left\{K(x) \mid K \in \mathbb{C}^{\prime}\right\} \\
& \left.=\sup \{K(x) \mid K \in \mathbb{C}, K \subseteq A\}=\underline{\operatorname{apr}}_{\mathbb{C}, \mathrm{InEx}}^{\prime}(A)\right)(x) .
\end{aligned}
$$

This completes the proof.

The model of $\mathrm{Li}$ et al. is incomparable with all other models for the logical connectives $\left(\mathscr{T}, \mathscr{I}_{\mathscr{T}}\right)$ with $\mathscr{T}$ left-continuous.

\subsection{Minimum t-norm and Kleene-Dienes implicator}

For the logical connectives $\left(\mathscr{T}_{M}, \mathscr{I}_{K D}\right)$, the model of $\mathrm{Li}$ et al. and Inuiguchi et al. are incomparable. Both models are also incomparable to the model of Wu et al., the model based on representation by levels and the intuitive extension. However, both models are comparable with the model of Feng et al. if the induced covering of $\mathbb{C}$ is used:

Proposition 15. Let $\mathbb{C}$ be a covering and $A \in \mathscr{F}(U)$, then for $\mathscr{T}$ the minimum t-norm and $\mathscr{I}$ the KleeneDienes implicator it holds that

(a) $\underline{\operatorname{apr}}_{\mathbb{C}, \mathrm{Fe}}^{\prime}(A) \subseteq \underline{\operatorname{apr}}{ }_{\mathrm{Cov}(\mathbb{C}), \mathrm{Li}}(A)$,

(b) $\underline{\operatorname{apr}}_{\mathbb{C}, \mathrm{Fe}}^{\prime}(A) \subseteq \underline{\operatorname{apr}} \underset{\mathrm{Cov}(\mathbb{C}), \operatorname{In}}{ }(A)$.

Proof. To prove (a), take $x \in U$. For $K_{x} \in \operatorname{Cov}(\mathbb{C})$ it holds that $K_{x}(x)=1$, so we have that

$$
\begin{aligned}
& \left.\underline{\operatorname{apr}}_{\mathrm{Cov}(\mathbb{C}), \mathrm{Li}}^{\prime}(A)\right)(x) \\
& \geq \min \left(K_{x}(x), \inf _{y \in U} \max \left(1-K_{x}(y), A(y)\right)\right) \\
& =\inf _{y \in U} \max \left(1-K_{x}(y), A(y)\right)=\left(\underline{\operatorname{apr}}_{\mathbb{C}, \mathrm{Fe}}^{\prime}(A)\right)(x) .
\end{aligned}
$$


The proof of (b) is analogous.

Note that it is necessary to use the induced covering of $\mathbb{C}$ in the models of Li et al. and Inuiguchi et al., since both models are incomparable with the model of Feng et al. if $\mathbb{C} \neq \operatorname{Cov}(\mathbb{C})$.

\section{Conclusion and Future Work}

We have discussed six different fuzzy covering based models. The models of $\mathrm{Li}$ et al. and Inuiguchi et al. coincide for $\left(\mathscr{T}, \mathscr{I}_{\mathscr{T}}\right)$ with $\mathscr{T}$ left-continuous. Moreover, we have studied which properties of the tight covering based rough set model still hold for the different models. Only the model of Li et al. combined with the connectives $\left(\mathscr{T}_{M}, \mathscr{I}_{K D}\right)$ is not very interesting from theoretical perspective.

Additionally, we have discussed interrelationships between the models. The model of $\mathrm{Wu}$ et al., the model based on representation by levels and the intuitive extension are always comparable. The models of $\mathrm{Li}$ et al. and Inuiguchi et al. are comparable with the intuitive extension for $\left(\mathscr{T}, \mathscr{I}_{\mathscr{T}}\right)$ and with the model of Feng et al. for $\left(\mathscr{T}_{M}, \mathscr{I}_{K D}\right)$ and $\operatorname{Cov}(\mathbb{C})$.

Future work includes the study of fuzzy extensions of the loose pair of covering based rough set model, as well as the research of new fuzzy covering based rough set models. Moreover, we are interested in the applicability of fuzzy covering based rough sets in feature selection [18].

\section{Acknowledgements}

Lynn D'eer has been supported by the Ghent University Special Research Fund. This work was partially supported by the Spanish Ministry of Science and Technology under the project TIN2011-28488 and the Andalusian Research Plans P11-TIC-7765 and P10-TIC-6858, and by project PYR-2014-8 of the Genil Program of CEI BioTic GRANADA.

\section{References}

[1] Z. Pawlak, Rough sets, International Journal of Computer and Information Sciences 11 (1982) 341-356

[2] W. Żakowski, Approximations in the space $(u, \pi)$, Demonstratio Mathematica 16 (1983) 761-769

[3] J.A. Pomykała, Approximation operations in approximation space, Bulletin de la Académie Polonaise des Sciences 35 (9-10) (1987) 653-662.

[4] Y.Y. Yao, B. Yao, Covering based rough sets approximations. Information Sciences 200 (2012) 91-107.

[5] L.A. Zadeh, Fuzzy sets, Information and Control 8 (1965) 338-353

[6] T.J. Li, Y. Leung, W.X. Zhang, Generalized fuzzy rough approximation operators based on fuzzy coverings, International Journal of Approximate Reasoning 48 (2008) 836-856
[7] T. Feng, S.P. Zhang, J.S. Mi, The reduction and fusion of fuzzy covering systems based on the evidence theory, International Journal of Approximate Reasoning 53 (2012) 87-103

[8] M. Inuiguchi and T. Tanino, New fuzzy rough sets based on certainty qualification, in $\mathrm{K}$. Pal et al. (eds.), Rough-Neural Computing, Springer, Berlin-Heidelberg (2003) 278-296

[9] M. Inuiguchi, Classification and approximation oriented fuzzy rough sets, in B. Bouchon-Meunier et al. (eds.), Proceedings of Information Processing and Managment of Uncertainty in KnowledgeBased Systems (2004) cd-rom

[10] M.F. Wu, H.H. Han and Y.F. Si, Properties and axiomatization of fuzzy rough sets based on fuzzy coverings, Proceedings of the 2012 International Conference on Machine Learning and Cybernetics (2012) 184-189

[11] I. Couso and D. Dubois, Rough sets, coverings and incomplete information, Fundamenta Informaticae 108 (2011) 223-247.

[12] T. Deng, Y. Chen, W. Xu and Q. Dai, A novel approach to fuzzy rough sets based on a fuzzy covering, Information Sciences 177 (11) (2007) 2308 2326

[13] D. Sánchez, M. Delgado, M.A. Vila, J. ChamorroMartínez, On a non-nested level-based representation of fuzziness, Fuzzy Sets and Systems 192 (2012) 159-175

[14] E. Turunen, Mathematics behind fuzzy logic, Physica-Verlag, Wurzburg (1999)

[15] C. Cornelis, M. De Cock, A.M. Radzikowska, Fuzzy rough sets: from theory into practice, in: Handbook of Granular Computing (W. Pedrycz, A. Skowron, V. Kreinovich, eds.), Wiley (2008) 533 552

[16] D. Dubois and H. Prade, Rough fuzzy sets and fuzzy rough sets, International Journal of General Systems 17 (1990) 191-209.

[17] D. Dubois and H. Prade, Putting fuzzy sets and rough sets together, in R. Słowiński, editor, Intelligent Decision Support - Handbook of Applications and Advances of the Rough Sets Theory, Kluwer Academic Publishers (1992) 203-232

[18] C. Cornelis, R. Jensen, G. Hurtado, D. Ślȩzak, Attribute selection with fuzzy decision reducts, Information Sciences, 180 (16) (2010) 3006-3020 